\title{
Substrate recognition by complement convertases revealed in the $\mathrm{C5}$-cobra venom factor complex
}

\author{
Nick S Laursen ${ }^{1}$, Kasper R Andersen ${ }^{1}$, \\ Ingke Braren², Edzard Spillner², \\ Lars Sottrup-Jensen ${ }^{1}$ and \\ Gregers R Andersen ${ }^{1, *}$
}

${ }^{1}$ Department of Molecular Biology, Aarhus University, Aarhus, Denmark and ${ }^{2}$ Institute of Biochemistry and Molecular Biology, Department of Chemistry, University of Hamburg, Hamburg, Germany

Complement acts as a danger-sensing system in the innate immune system, and its activation initiates a strong inflammatory response and cleavage of the proteins $\mathrm{C3}$ and C5 by proteolytic enzymes, the convertases. These contain a non-catalytic substrate contacting subunit (C3b or C4b) in complex with a protease subunit (Bb or $\mathrm{C} 2 \mathrm{a})$. We determined the crystal structures of the $c 3 b$ homologue cobra venom factor (CVF) in complex with $\mathrm{C5}$, and in complex with C5 and the inhibitor SSL7 at $4.3 \AA$ resolution. The structures reveal a parallel two-point attachment between C5 and CVF, where the presence of SSL7 only slightly affects the C5-CVF interface, explaining the IgA dependence for SSL7-mediated inhibition of C5 cleavage. CVF functions as a relatively rigid binding scaffold inducing a conformational change in $\mathrm{C5}$, which positions its cleavage site in proximity to the serine protease $\mathrm{Bb}$. A general model for substrate recognition by the convertases is presented based on the C5-CVF and $\mathrm{C} 3 \mathrm{~b}-\mathrm{Bb}-\mathrm{SCIN}$ structures. Prior knowledge concerning interactions between the endogenous convertases and their substrates is rationalized by this model.

The EMBO Journal (2011) 30, 606-616. doi:10.1038/

emboj.2010.341; Published online 7 January 2011

Subject Categories: immunology; structural biology

Keywords: complement system; immunology; protein complex; proteolytic regulation; structural biology

\section{Introduction}

Activation of the proteolytic complement cascade triggers cleavage of the homologous $185-200 \mathrm{kDa}$ proteins C3, C4, and C5 (Supplementary Figure S1). Their proteolytic fragments mediate enhanced phagocytosis and pathogen lysis, clearance of immune complexes and apoptotic cells, inflammation and promote adaptive immune responses (Walport, 2001; Gasque, 2004). Three activation pathways converge at the formation of $\mathrm{C} 3$ convertases, cleaving C3 into C3a and $\mathrm{C} 3 \mathrm{~b}$. The classical pathway (CP) is activated by surface-

\footnotetext{
*Corresponding author. Department of Molecular Biology, Aarhus University, Gustav Wieds Vej 10C, Aarhus DK-8000, Denmark. Tel: + 45894250 24; Fax: + 4586123178 ;

E-mail: gra@mb.au.dk
}

Received: 26 August 2010; accepted: 26 November 2010; published online: 7 January 2011 bound immune complexes, resulting in cleavage of $\mathrm{C} 4$ and thereby generation of $\mathrm{C} 4 \mathrm{~b}$ (Figure 1A). Proteolysis of $\mathrm{C} 4$ is also triggered via recognition of microbial carbohydrates in the lectin pathway (LP). C4b combines with C2 and subsequent cleavage of $\mathrm{C} 2$ to $\mathrm{C} 2$ a results in generation of the $\mathrm{LP} / \mathrm{CP}$ C3 convertase C4b2a. (C4b2a is the widely used short-hand nomenclature for the $\mathrm{C} 4 \mathrm{~b}-\mathrm{C} 2 \mathrm{a}$ complex in the complement field, and similar nomenclature is used for the remaining complexes in the following). The alternative pathway (AP) $\mathrm{C} 3$ convertase $\mathrm{C} 3 \mathrm{bBb}$ is formed when factor $\mathrm{B}(\mathrm{fB})$ combines with $\mathrm{C} 3 \mathrm{~b}$, after which $\mathrm{fB}$ is activated by factor $\mathrm{D}$ (fD) (Figure 1A). Normally the C3 convertases are surface anchored, as an internal thioester present in both nascent $\mathrm{C} 3 \mathrm{~b}$ and $\mathrm{C} 4 \mathrm{~b}$ can react with a surface nucleophile. However, a fluid-phase AP C3 convertase $\mathrm{C} 3\left(\mathrm{H}_{2} \mathrm{O}\right) \mathrm{Bb}$ can also assemble after spontaneous hydrolysis of the $\mathrm{C} 3$ thioester and initiate the AP (Pangburn and Muller-Eberhard, 1983). Both the AP and CP C3 convertases may recruit an additional C3b molecule (Takata et al, 1987; Kinoshita et al, 1988; Pangburn and Rawal, 2002) to form a C5 convertase (C3bBb3b or $\mathrm{C} 4 \mathrm{~b} 2 \mathrm{a} 3 \mathrm{~b}$ ), which cleaves $\mathrm{C} 5$ to generate the large fragment $\mathrm{C} 5 \mathrm{~b}$ and the anaphylatoxin C5a (Figure 1A). Cobra venom factor (CVF) is a C3b homologue found in the venom of snakes from the genus Naja. Together with Bb, CVF assembles into a highly stable fluid phase convertase CVFBb cleaving both C3 and C5 (Vogel and Fritzinger, 2010). The release of C3a and C5a increases vascular permeability and blood flow, thereby promoting further transport of other snake venom components (Vogel and Fritzinger, 2010). Staphylococcus aureus secretes various proteins, which manipulate the functions of the complement system (Geisbrecht, 2008). One of these, SSL7, inhibits complement-mediated haemolytic and bactericidal activity by binding to $\mathrm{C} 5$ through its C-terminal $\beta$-grasp domain (Laursen et al, 2010). SSL7 is bifunctional and binds IgA through its N-terminal OB domain blocking the recognition of IgA by Fc $\alpha$ RI (Ramsland et al, 2007). SSL7 binds to C5 at $>70 \AA$ from the cleavage site in C5 and possibly interferes with $\mathrm{C} 5$ recognition by convertases through IgA-dependent steric hindrance (Laursen et al, 2010).

To provide insight into the substrate recognition by the complement convertases, we studied the CVF-C5 complex. In contrast to $\mathrm{C} 3 \mathrm{~b}, \mathrm{CVF}$ binds with high affinity to $\mathrm{C} 5$, which allowed us to crystallize the C5-CVF complex and determine its structure. Furthermore, to fully establish the mechanism of inhibition by SSL7, we determined the structure of the SSL7-C5-CVF complex. The structures reveal that two distinct surface areas on both C5 and CVF mediate complex formation. CVF binding is shown to induce conformational changes in $\mathrm{C} 5$, which changes the position of the convertase cleavage site in C5, while complex formation with SSL7 results in a minor disturbance of the C5-CVF interface. The C5-CVF structure validates prior data concerning interactions between the endogenous convertases and their substrates and suggests a preferred orientation of surfacebound convertases. 
A

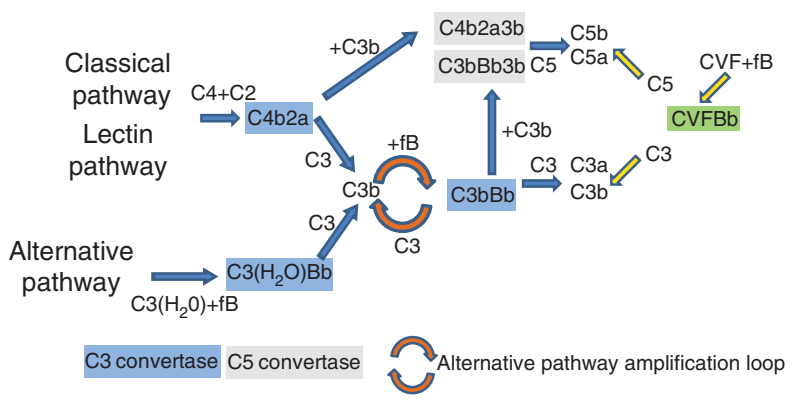

B

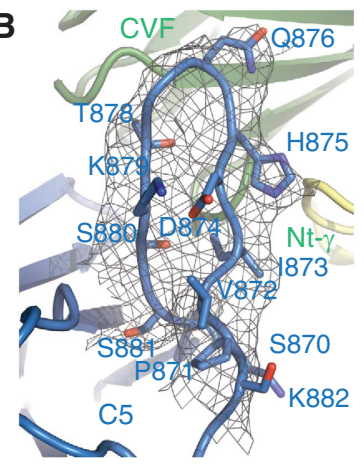

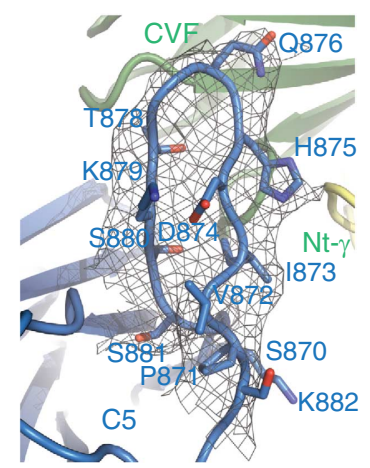

C

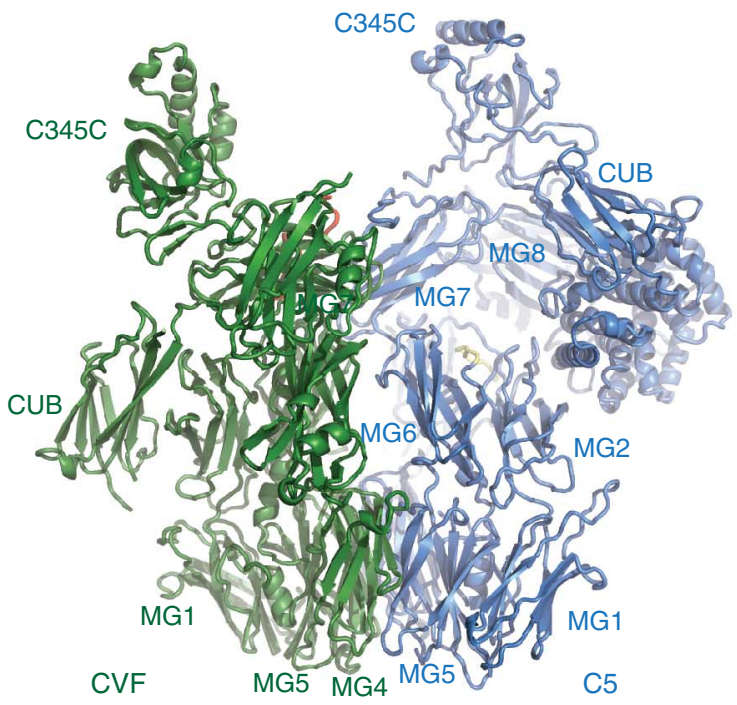

D

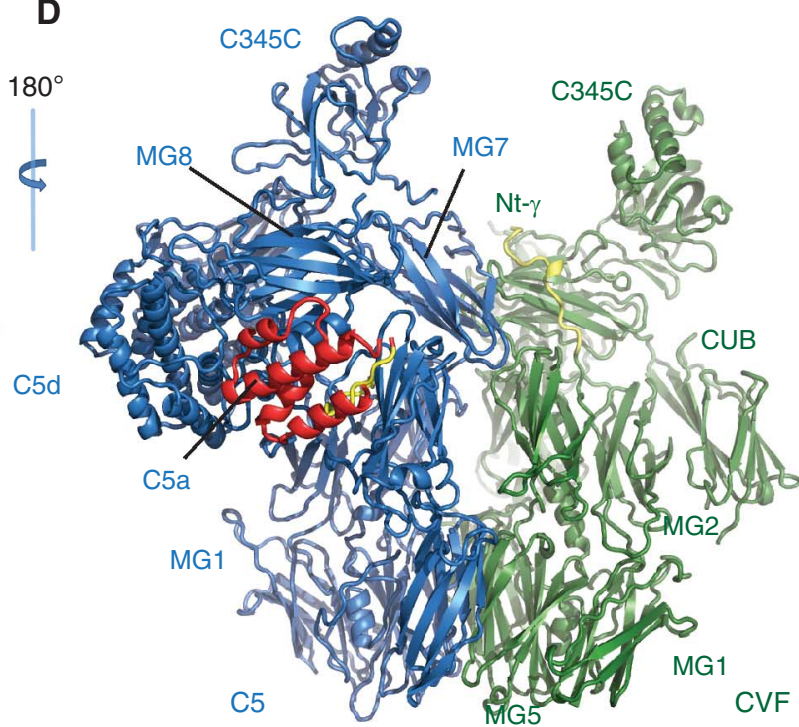

Figure 1 Complement activation and the structure of the C5-CVF complex. (A) The three activation pathways of complement (left) induce the assembly of the proteolytic C3 convertases and, after additional C3 cleavage, the C5 convertases. Factor B, C4, and C2 are cleaved by the pathway-specific serine proteases factor D, MASP-2, and C1s. CVFBb (right, green box) cleaves both C3 and C5. Abbreviations for the complexes: C4b2a, C4b-C2a complex; C3bBb, C3b-Bb complex; C4b2a3b, C4b-C2a-C3b complex; C3bBb3b, C3b-Bb-C3b complex; CVFBb, CVF-Bb complex; $\mathrm{C} 3\left(\mathrm{H}_{2} \mathrm{O}\right) \mathrm{Bb}, \mathrm{C} 3\left(\mathrm{H}_{2} \mathrm{O}\right)-\mathrm{Bb}$ complex. (B) Stereo view of omit non-averaged $2 \mathrm{mF}_{\mathrm{o}}-\mathrm{DF}_{\mathrm{c}}$ electron densities contoured at $1 \sigma$ around the loop Ser870-Lys882 in the C5 MG7 domain, which becomes ordered in the CVF complex in contrast to free C5. For residues with side chains shown, all atoms were omitted for map calculation. (C, D) Cartoon representation of C5 (C5a red, future Nt- $\alpha^{\prime}$ yellow, blue otherwise) in complex with CVF (Nt- $\gamma$ yellow, otherwise green). This colouring scheme is used throughout unless otherwise noted. See also Supplementary Figure S1 for definition of domains and chains.

\section{Results}

\section{The structure of C5-CVF}

The endogenous C3 and C5 convertases are very large unstable macromolecular complexes with a lifetime of a few minutes and four out of five convertases are surface anchored. Crystallization of these convertases either alone or in complex with their substrates is therefore a challenging task. As an alternative we used CVF, which forms a very stable and soluble complex with C5 ( $K_{\mathrm{d}}$ of $42 \mathrm{~nm}$ ) (Rawal and Pangburn, 2000). We determined the crystal structures of the C5-CVF and the SSL7-C5-CVF complexes at $4.3 \AA$ resolution (Figures 1 and 2; Table I; Supplementary Tables I and II). Despite the low resolution of the structures, the main-chain tracing is generally reliable due to the starting models of $\mathrm{C} 5$, C5-SSL7, and CVF determined at 3.1, 3.6, and 2.2 $\AA$, respectively, used for structure determination (Figures $1 \mathrm{~B}$ and $2 \mathrm{~A}$ ). Rigid body refinement and grouped B-factor refinement of the starting models reduced the $R_{\text {free }}$ to $29-30 \%$ without any manual rebuilding. More detailed conformational changes were then modelled in $2 \mathrm{mF}_{\mathrm{o}}-\mathrm{DF}_{\mathrm{c}}$ electron density maps using a database of known structures for model building (Jones et al, 1991) before further positional refinement. Except for a few regions differing due to crystal packing, two-fold averaged $2 \mathrm{mF}_{\mathrm{o}}-\mathrm{DF}_{\mathrm{c}}$ electron density maps were used for rebuilding of both complexes. For this reason, the assignment of regions involved in intermolecular contacts within the C5-CVF and the SSL7-C5-CVF complexes can be carried out with confidence. The modelled position of side chains must be judged with caution due to the low resolution of the diffraction data, and detailed intermolecular interactions such as hydrogen bonds and electrostatic interactions are difficult to assign unambiguously.

C5 and CVF are arranged in a head-to-head manner and form a complex of dimensions $160 \times 154 \times 90 \AA$ (Figure 1C and D) with the long axis of the two molecules aligned roughly in parallel. Two widely separated regions on C5 mediate binding to $\mathrm{CVF}$ and vice versa (Figure 3A and B; Supplementary Figure S2A and C. Note that prepro-numbering is used for all proteins.) Although many charged side 


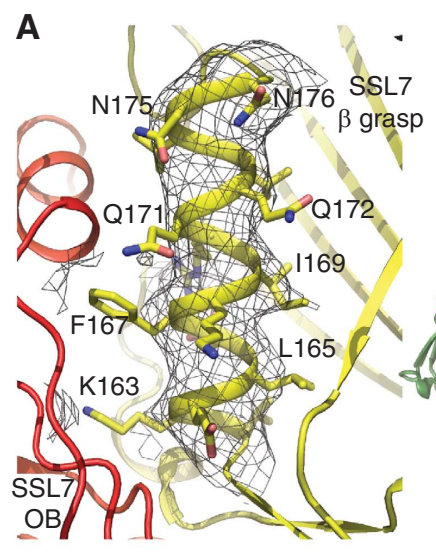

\section{B}

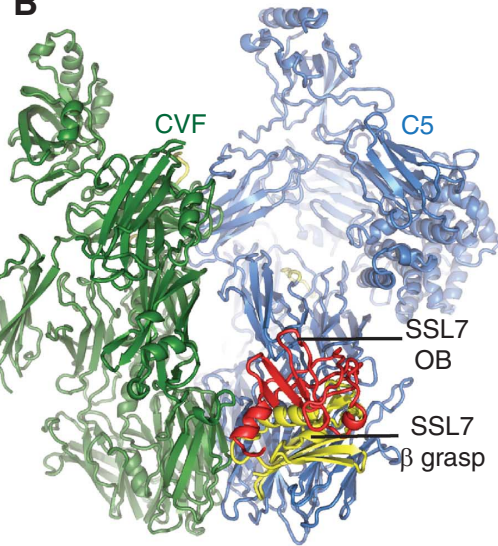

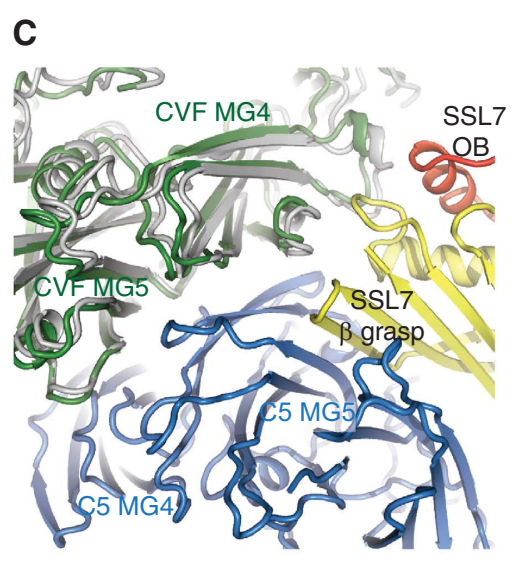

Figure 2 The structure of the SSL7-C5-CVF complex. (A) Omit non-averaged $2 \mathrm{mF}_{\mathrm{o}}-\mathrm{DF}_{\mathrm{c}}$ electron densities contoured at $1 \sigma$ from the SSL7-C5CVF complex around the large helix in the OB domain of SSL7. For residues with side chains shown, all atoms were omitted for map calculation. (B) SSL7 binds through its $\beta$-grasp domain (yellow) to the C5 MG5 domain, but the $\beta$-grasp and the OB domain (red) also appear to interact with the CVF MG4 domain. (C) Comparison of the position of CVF (grey) in its complex with C5 (blue), and of CVF (green) in complex with SSL7-C5.

Table I Data collection and refinement statistics

\begin{tabular}{lcc}
\hline & C5-CVF & SSL7-C5-CVF \\
\hline $\begin{array}{l}\text { Data collection } \\
\text { Space group }\end{array}$ & $\mathrm{P} 222_{1}$ & $\mathrm{P} 222_{1}$ \\
Cell dimensions & $176.52,179.20$, & $163.65,181.96$, \\
$\quad a, b, c(\AA)$ & 389.69 & 392.78 \\
& $50-4.3(4.43-4.30)^{\mathrm{a}}$ & $50-4.3(4.42-4.30)$ \\
Resolution $(\AA)$ & $14.3(62.5)$ & $14.3(52.5)$ \\
$R_{\text {sym }}$ & $10.9(3.1)$ & $11.4(3.7)$ \\
$I / \sigma I$ & $94.2(96.0)$ & $96.8(98.6)$ \\
Completeness $(\%)$ & $5.6(5.6)$ & $5.1(5.1)$ \\
Redundancy & & \\
& & \\
Refinement & $49.5-4.3$ & $49.2-4.3$ \\
Resolution $(\AA)$ & 79835 & 77924 \\
No. of reflections & $22.6 / 26.3$ & $22.4 / 25.5$ \\
$R_{\text {work }} / R_{\text {free }}$ & & \\
No. of atoms & 45184 & 48212 \\
Protein & 140 & 140 \\
Glycosylations & & 171.40 \\
B factors & 196.33 & 291.3 \\
Protein & 336.72 & \\
Glycosylations & & 0.012 \\
r.m.S. deviations & 0.012 & 1.63 \\
Bond lengths $(\AA)$ & 1.56 & \\
Bond angles $(\mathrm{deg})$ &
\end{tabular}

${ }^{\mathrm{a}}$ Values in parentheses are for the highest-resolution shell. One crystal was used for each dataset. Further details of statistics for data collection and refinement are found in Supplementary Tables I and II.

chains are found at the intermolecular interface, the electrostatic potentials of the two proteins (Figure $3 \mathrm{~A}$ and $\mathrm{B}$ ) do not suggest ionic interactions to be the driving force for the intermolecular interaction. In agreement with this, both the C5-CVF and the SSL7-C5-CVF complex were crystallized under similar solution conditions with high ionic strength. The largest of the two interfaces between C5 and CVF involves contacts between the MG4 and MG5 domains from both proteins (Figure 3C and D; Supplementary Figure S3A, B, E, and F) and has a calculated area of $1350 \AA^{2}$ (Supplementary Table III). We will refer to this interface as the MG4MG5 interface. Residues within the C5 MG4 domain interact exclusively with the CVF MG5 domain, while the C5 MG5 domain interacts mainly with CVF MG4 supplemented with a few contacts to CVF MG5 (Figure 3C and D; Supplementary Figure S3A and B). In C5, residues within the regions Ser419Pro425, Thr470-Ile485, and Asp520-Asn527 are located in the vicinity of CVF residues Ser386-Thr389, Ile399-Leu404, Thr450-Lys467, and Arg498-Asn507. The MG4-MG5 interface is rather flat by nature and with few conformational changes in the participating regions compared with the structures of $\mathrm{C} 5$ and $\mathrm{CVF}$ alone. The other contact area, referred to as the MG7 interface, is formed between the C5 MG7 domain and the CVF MG6 and MG7 domains (Figure 3E and F; Supplementary Figure S3C, D, G, and H). It has a calculated area of $830 \AA^{2}$ and appears to be more intricate than the MG4-MG5 interface. A hydrophobic core within the interface is formed by C5 Met853, Trp917, and Phe918 together with CVF residues Ile556, Met558, Val813, Leu904, and Trp905 (Figure 3E and F; Supplementary Figure S3C and D). The MG7 loop 871-882-disordered in the structure of free C5-forms an ordered loop resulting in putative contacts with CVF Tyr845, Gln901, and Glu902 (Figure 1B and 3E, F; Supplementary Figures S2 and S3). Contacts between this C5 loop and CVF are consistent with the decrease in CVFBbmediated C5 cleavage after deletion of C5 Ser881-Gln886 (Low et al, 1999). Many C5 residues engaging in putative CVF interactions are well conserved in C5 and C3 (Supplementary Figure S2A and B), both substrates for CVFBb. CVF residues appearing to be in contact with $\mathrm{C} 5$, especially those presumably interacting with the C5 MG7 domain, are in general less conserved upon alignment with C3 (Supplementary Figure S2C), perhaps explaining the much higher affinity of CVF for C5 compared with C3b (Rawal and Pangburn, 2000). The conformation of C5b is apparently similar to that of C3b (Hadders et al, 2010), suggesting that in $\mathrm{C} 5 \mathrm{~b}$ the MG7 domain is located very differently relative to the C5 MG4-MG5 domains, and that the C5d and CUB domains are released from the MG8 domains and become exposed in C5b. Hence, C5 bound to CVF is kept in a conformation much closer to that of unbound $\mathrm{C} 5$ compared with $\mathrm{C} 3 \mathrm{~b} / \mathrm{C} 5 \mathrm{~b}$, and the conformational change 

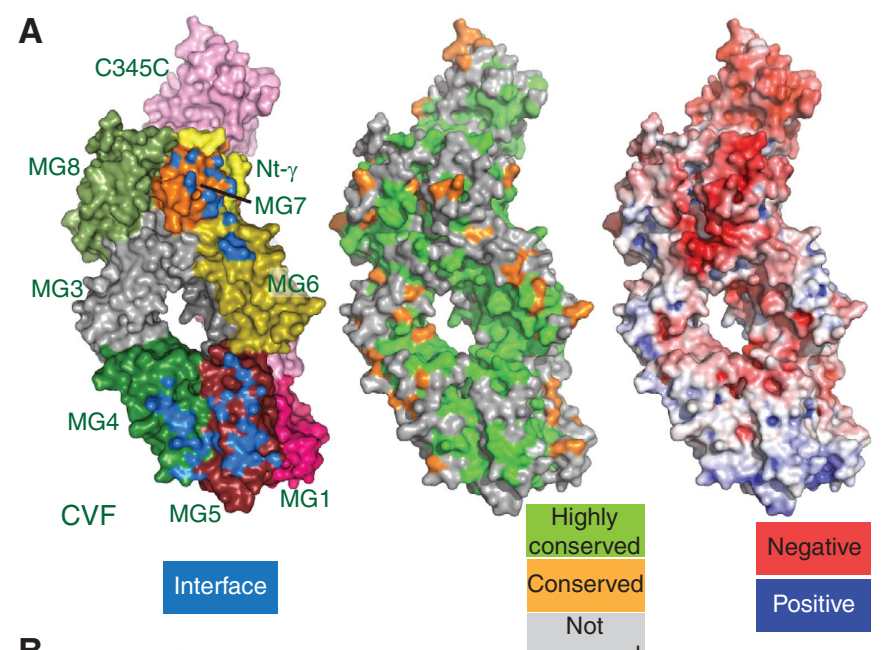

B
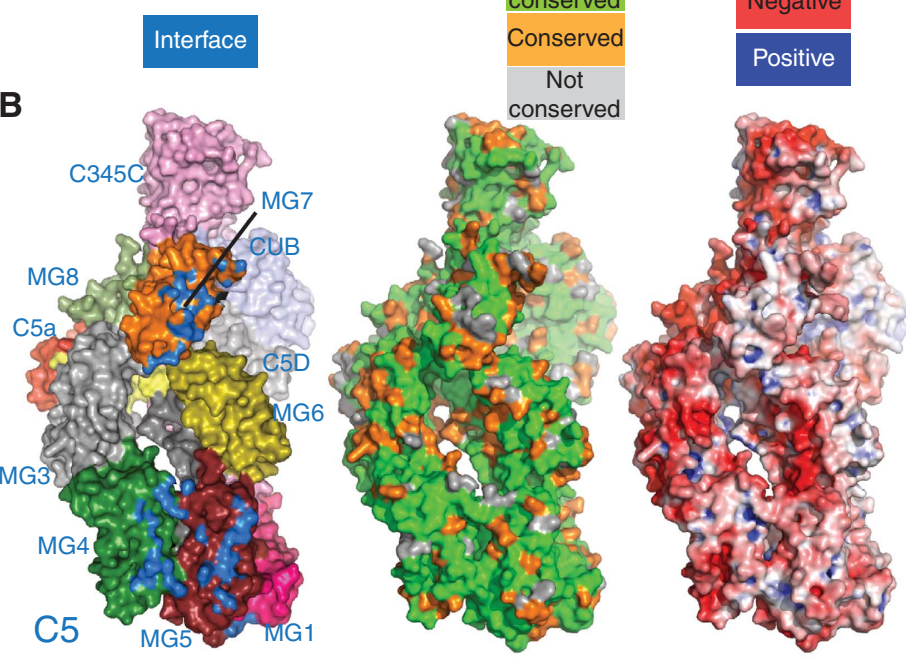

C

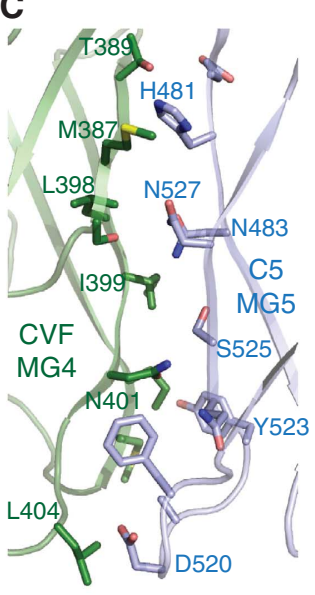

D

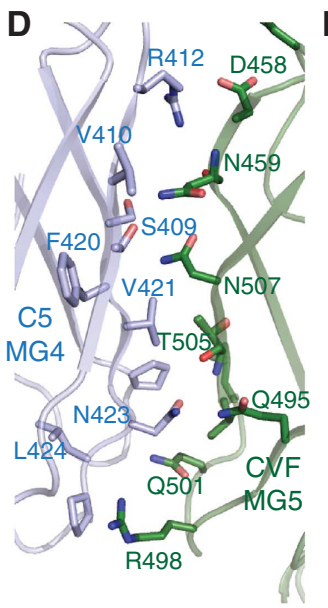

E

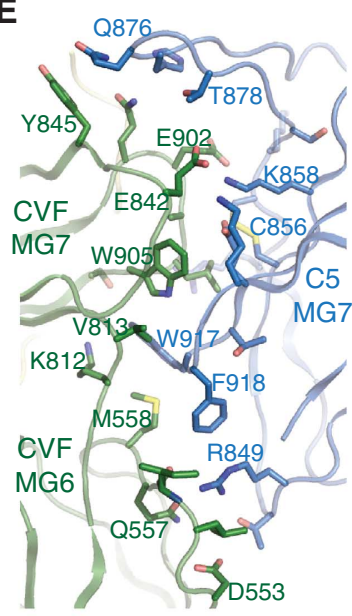

$\mathbf{F}$

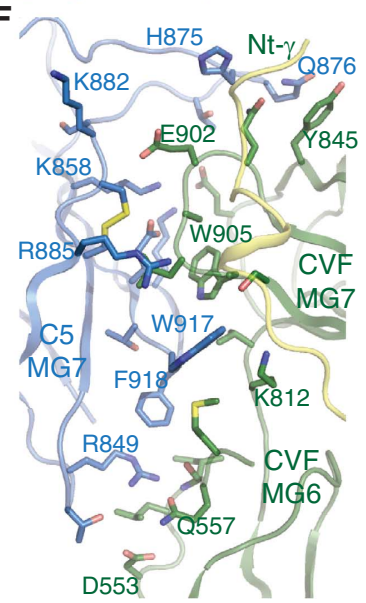

Figure 3 C5-CVF interactions. (A) Surface representation of CVF with its structural domains individually coloured and residues contacting C5 coloured blue (left panel), CVF coloured according to conservation within CVF and mammalian C3 sequences (middle panel, see also Supplementary Figure S2C), and CVF with the electrostatic potential mapped to the surface (right panel). The view is related to that of Figure $1 \mathrm{C}$ by a $90^{\circ}$ rotation around a vertical axis. (B) As in panel A, but with C5 residues interacting with CVF coloured blue (left), C5 coloured according to conservation (middle, see also Supplementary Figure S2A), and the electrostatic potential mapped on the surface of C5 (right). The view is related to that of panel A by a $180^{\circ}$ rotation around a vertical axis. (C, D) Close-up of the MG4-MG5 interface between C5 and CVF in two orientations. (E, F) Details of the C5-CVF MG7 interface in two orientations.

between free and bound C5 is likely to be reversible. Importantly, CVF cannot interact with the MG4, MG5, and MG7 domains in the product C5b in the same manner as with the substrate $\mathrm{C} 5$, providing a mechanism for distinguishing substrate from product.

\section{Conformational changes in $\mathrm{C5}$}

Complex formation with C5 has little influence on the conformation of CVF compared with either free CVF (Krishnan et al, 2009) or CVF in complex with $\mathrm{fB}$ (Janssen et al, 2009). Only the C-terminal C345C and the CUB domains have a slightly variable orientation relative to the remaining $\mathrm{CVF}$ molecule. But these discrepancies may easily be caused by differences in crystal packing interactions and the presence of factor B in the CVFB complex, and neither of the two domains are in direct contact with C5. Likewise, the conformation of C5-bound CVF is also close to that of $\mathrm{C} 3 \mathrm{~b}$ alone (Supplementary Figure S4) or $\mathrm{C} 3 \mathrm{~b}$ in complex with $\mathrm{Bb}$ and the bacterial convertase inhibitor SCIN (Rooijakkers et al, 2009). CVF residues $20-1473$ can be superimposed onto CVF from its complex with $\mathrm{fB}$ with an r.m.s.d. of $1.34 \AA$ over 876 $\mathrm{C}_{\alpha}$ atoms, and superimposed with the structure of free CVF with an r.m.s.d. of $1.57 \AA$ over 776 atoms. CVF residues 201473 from the C5-CVF complex can also be overlaid with C3b from its complex with $\mathrm{Bb}$ and SCIN with an r.m.s.d. of $1 \AA$ for $824 \mathrm{C}_{\alpha}$ atoms. Hence, CVF seemingly acts as a relatively rigid scaffold for binding of both $\mathrm{Bb}$ and $\mathrm{C} 5$ in CVFBb. In contrast, C5 undergoes a significant overall conformational change upon CVF binding (Figure 4A and B; Supplementary Figure S5; Supplementary Animation 1). Residues 18-1510 from the structure of free $\mathrm{C} 5$ can be overlaid onto $\mathrm{C} 5$ from the CVF complex with an r.m.s.d. of $2.45 \AA$ over $1307 \mathrm{C}_{\alpha}$ atoms. The conformational change within $\mathrm{C} 5$ can roughly be described as a concerted movement, with the C5a, MG7, CUB, C5d, and MG8 domains rotating by $18^{\circ}$ relative to the MG1, MG4, and MG5 domains, a second structural entity that also remains largely unaffected compared with free C5. Located between these two structural entities, the MG3 domain rotates by $23^{\circ}$ relative to either entity, while the domain pair MG2-MG6 turns by $10-12^{\circ}$. Separate from these effects is the major rearrangement that places the $\mathrm{C} 5 \mathrm{C} 345 \mathrm{C}$ domain in a C3-like position (discussed below). The conformational rearrangement significantly changes the interdomain interactions of one end of the MG3 domain. In unbound C5 this end is 
A

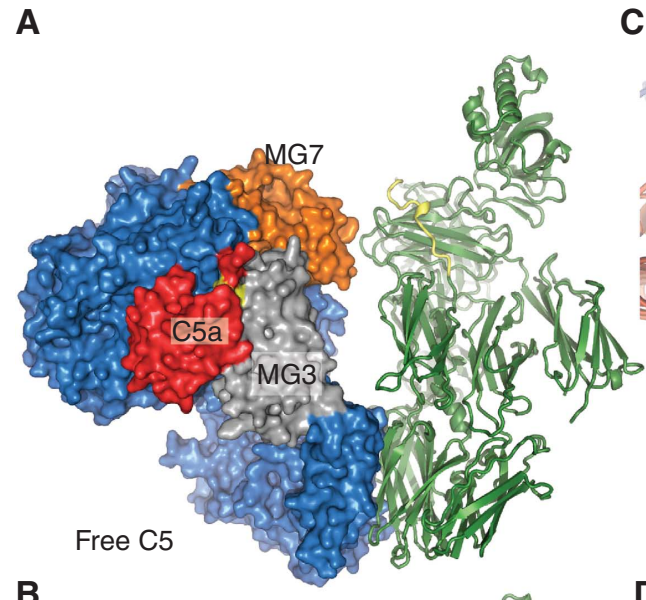

B

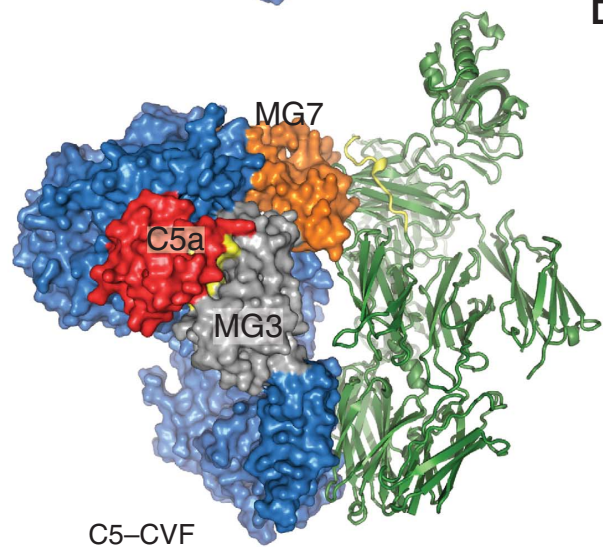

C

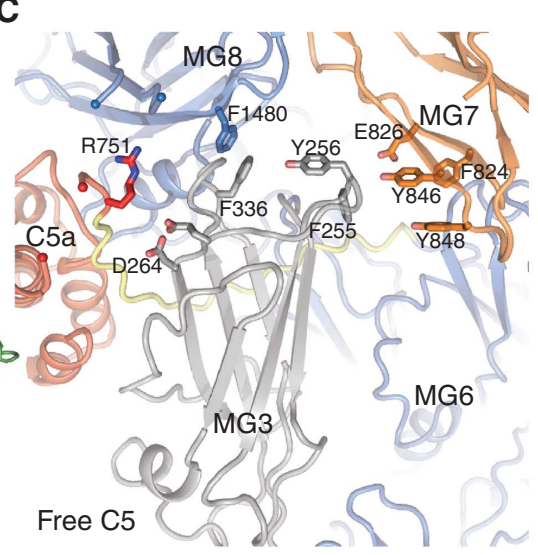

D

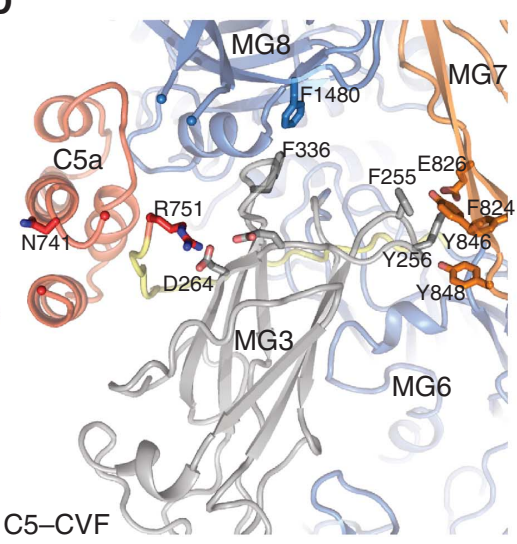

E

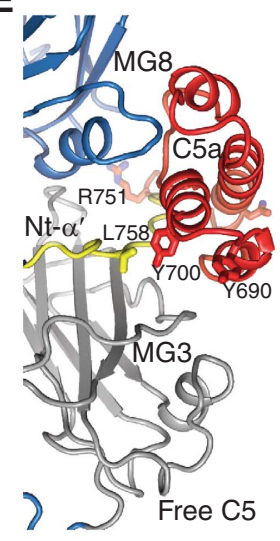

$\mathbf{F}$

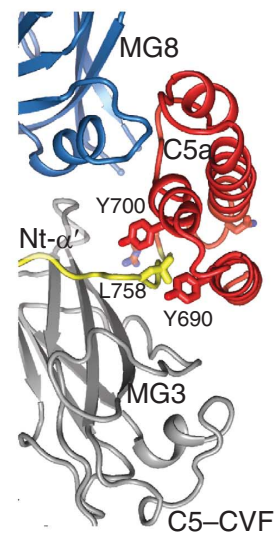

Figure 4 Conformational changes in C5 upon binding to CVF. (A) Surface representation of free C5 with the MG3 and MG7 domains coloured grey and orange, respectively, and CVF shown in cartoon for comparison. Free C5 was placed by superposition of its MG4 and MG5 domains with the same domains in C5-CVF. (B) As in panel A, but for the C5-CVF complex. The C5 C345C domain is not shown in panels A and B, as its position in free C5 is governed by crystal packing. (C) Close-up of the interaction between the MG3 and MG8 domains in free C5. (D) In the CVF-bound state of C5 the MG3 domain is shifted towards the MG7 domain. (E) The future $\mathrm{Nt}-\alpha^{\prime}$ chain (yellow) is sandwiched between C5a and the MG3 domain in free C5. (F) In the CVF-bound state of C5 the rotation of the MG3 domain induces conformational changes in Nt- $\alpha^{\prime}$. The two states of C5 in panels C-F were superimposed through the CUB, C5d, and MG8 domains.

associated with the MG8 domain, but in C5-CVF it instead appears to interact with the MG7 domain (Figure 4C and D). The movement of the MG3 domain also changes the position of the future $\mathrm{Nt}-\alpha^{\prime}$ region, as this is sandwiched between the C5a and the MG3 domain (Figure 4E and F).

\section{The structure of the SSL7-C5-CVF complex}

The $S$. aureus protein SSL7 binds with high affinity to IgA and $\mathrm{C} 5$, thereby inhibiting the binding of IgA to the Fc $\alpha \mathrm{RI}$ receptor, C5a generation, and C5-mediated serum killing of Escherichia coli (Langley et al, 2005; Laursen et al, 2010). We earlier showed that efficient SSL7-mediated inhibition of C5 cleavage by CVFBb required the presence of the SSL7-binding IgA Fc moiety, and suggested that this was due to steric hindrance preventing convertase binding to C5 (Laursen et al, 2010). To confirm this assumption, we determined the crystal structure of the SSL7-C5-CVF complex. As in C5-SSL7 (Laursen et al, 2010), SSL7 recognizes the C5-CVF complex by $\beta$-sheet pairing between its $\mathrm{C}$-terminal $\beta$-grasp domain and the C5 MG5 domain, while the N-terminal OB domain of SSL7 is free to bind IgA. Within the SSL7-C5-CVF complex, both SSL7 domains apparently interact with the MG4 domain of CVF (Figure 2B and C). This causes a slight rotation of the CVF MG4 domain away from C5 MG5, which has little consequence for the putative contacts between the C5 MG4 and MG7 domains to CVF. This perturbation, however, probably explains the reduced affinity of immobilized SSL7-C5 for CVF (Supplementary Figure S6B) in a surface plasmon resonance (SPR) experiment compared with measurements of immobilized C5 (Rawal and Pangburn, 2000) and the slight inhibition of CVFBb-mediated C5 cleavage exerted by SSL7 alone (Laursen et al, 2010). Docking of IgA onto the SSL7-C5-CVF complex demonstrates massive steric hindrance between IgA and the CVF MG3 and MG4 domains (Supplementary Figure S7A). C5 and IgA bind to SSL7 with $K_{\mathrm{d}}$ values of 6 and $1 \mathrm{~nm}$, respectively, and a trimeric SSL7-C5IgA complex can be isolated (Ramsland et al, 2007; Laursen et al, 2010). Considering the in vivo concentrations of C5 and IgA, SSL7 most likely permanently binds both proteins simultaneously (Laursen et al, 2010). Although SSL7 alone inhibits $\mathrm{C} 5$ cleavage by $\mathrm{CVFBb}$ and the endogenous $\mathrm{C5}$ convertases to some degree, full inhibition is only obtained in the presence of IgA (Bestebroer et al, 2010; Laursen et al, 2010). This is confirmed by our structure and SPR experiments indicating that although SSL7 weakens the binding of CVF to C5, it does not prevent the complex formation, and SSL7 only marginally influences the interaction between C3b and C5 (Supplementary Figure S6).

In summary, our structures show how $\mathrm{C} 5$ and CVF interact in a head-to-head manner with the long axis of both proteins 
A
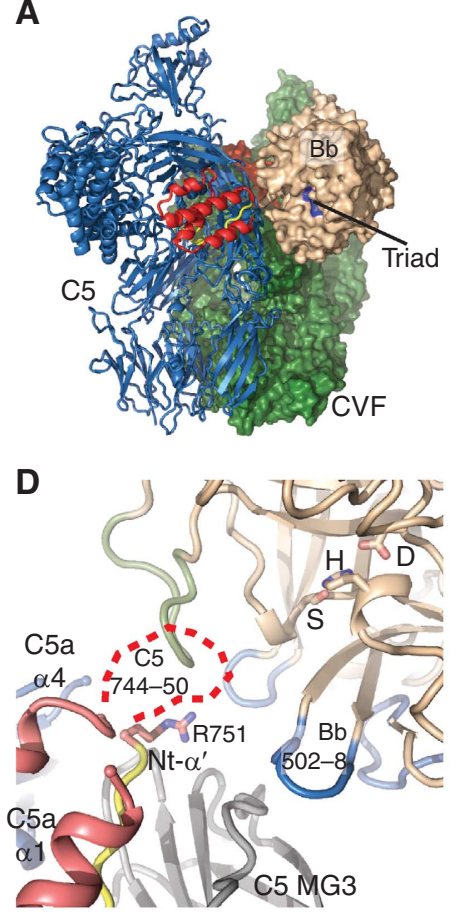

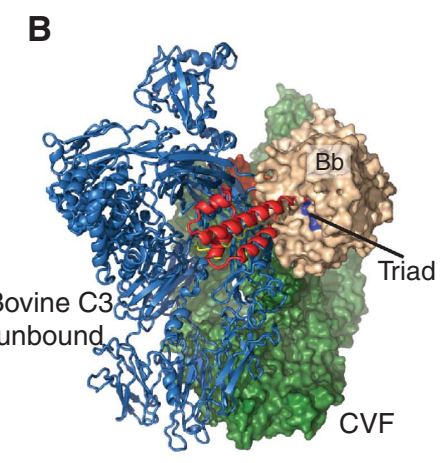

E

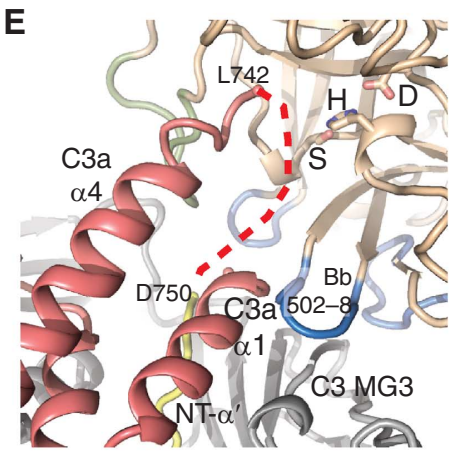

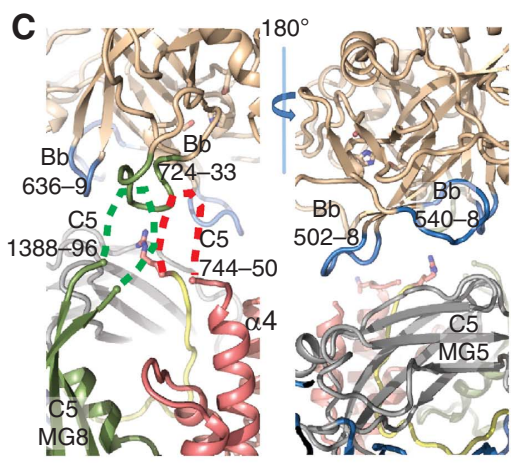

$\mathbf{F}$

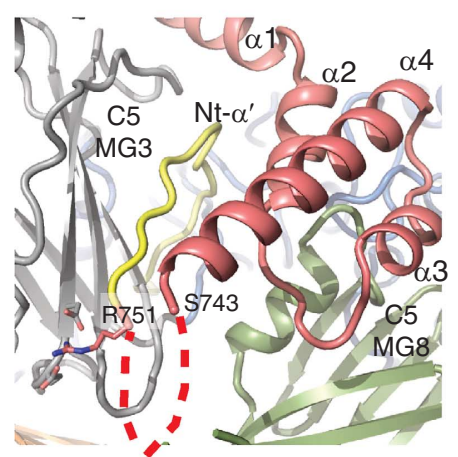

Figure 5 Models for substrate-CVFBb complexes. (A) C5 (blue cartoon, C5a red) in complex with CVF (green surface) and Bb (sand-coloured surface) bound to CVF. The blue area on Bb marks the catalytic triad cleaving C5 Arg751-Leu752. (B) As in panel A, but with C3 taking the position of $\mathrm{C} 5$. This conformation of bovine $\mathrm{C} 3$ has a three-dimensional arrangement of the CVF contacting domains resembling that of $\mathrm{C} 5$ bound to CVF (Supplementary Figure S5C and D). (C) Three surface-exposed loops (blue) in Bb are located close to the C5 MG3 domain (grey) and C5a (red), while a fourth loop (green) potentially interacts with the disordered loop (green dashed line, residues 1388-1396) in C5 MG8 (green) or the disordered C5a loop (red dashed line, residues 744-750). (D) Close-up of the suggested C5-Bb interface with C5a in red and the future Nt- $\alpha^{\prime}$ in yellow. In Bb the catalytic triad side chains are labelled $\mathrm{D}, \mathrm{H}$, and S. (E) As in panel $\mathbf{D}$, but with the docked C3 molecule pointing the cleaved bond Arg746-Ser747 further towards the catalytic site. (F) Model suggesting how relocation of $\alpha$ helix 1 in C5a could allow Nt- $\alpha^{\prime}$ to undergo a conformational change.

aligned approximately in parallel. The overall conformation of CVF is not significantly affected by interaction with the substrate, while a conformational change in C5 significantly changes the relative orientation of two surface areas in contact with CVF compared with free C5. Binding of SSL7 to the C5-CVF complex leads to a minor perturbation of the interaction between $\mathrm{C} 5$ and CVF, which is in agreement with the partial inhibition of C5 cleavage by SSL7 in the absence of IgA.

\section{Substrate recognition by $\mathrm{CVFBb}$}

By a simple superposition of CVF from the C5-CVF complex with C3b from the C3b-Bb-SCIN complex (Rooijakkers et al, 2009), we have created a model reflecting how C5 and by homology C3 are recognized by CVFBb (Figure 5; Supplementary Figure S5). In the $\mathrm{C} 5-\mathrm{CVFBb}$ model, the peptide bond cleaved in C5 between Arg751 and Leu752 is located $19 \AA$ away from its expected location in the catalytic site of $\mathrm{Bb}$ (Figure $5 \mathrm{D}$ ), suggesting conformational changes in $\mathrm{C} 5$ and $\mathrm{CVFBb}$ relative to our model. The flexibility of the CVF $\mathrm{C} 345 \mathrm{C}$ domain could contribute to bringing the $\mathrm{Bb}$ catalytic site to the substrate cleavage site as suggested (Rooijakkers et al, 2009). However, in the model, Bb loop regions flanking the entrance to the catalytic site are already within $5 \AA$ of C5 (Figure 5C) and therefore large-scale rigid body movement (e.g., larger than $5 \AA$ ) of $\mathrm{Bb}$ appears to be not required. As an alternative, we suggest that the local conformational changes in C5 residues 741-769 may be impor- tant for accommodating C5 Arg751 in the Bb active site. In C3, $\alpha$ helix 4 in C3a is longer than in C5 (Figure 5E), and elongating this by $1-2$ turns in C5 would significantly reduce the distance of residues upstream of Arg751 to the catalytic site. However, steric hindrance still appears to prevent C5 Arg751 from reaching the catalytic site, as residues Met754-Leu758 are sandwiched between C5a $\alpha$ helix 1 and the MG3 domain (Figure 4F). Such hindrance could be relieved if the helix is detached from the three other helices in C5a (Figure 5F) as recently observed in free C5a (Cook et al, 2010). The suggested local conformational changes in $\mathrm{C} 5$ could be triggered by contacts with $\mathrm{Bb}$, which might explain why $\mathrm{C} 5 \mathrm{~b}$, in contrast to $\mathrm{C} 3 \mathrm{~b}$, cannot be generated by less-specific proteases like trypsin (Fredslund et al, 2008).

As CVFBb also cleaves C3 (Vogel and Fritzinger, 2010), C3 is likely to adopt a conformation closely related to that of C5 in complex with CVF. Remarkably, a conformation of bovine C3 strongly resembling that of C5 bound to CVF has apparently been stabilized by packing in crystals of bovine $\mathrm{C} 3$ (Fredslund et al, 2006). A second copy of bovine C3 and human C3 (Janssen et al, 2005) does not adopt a conformation resembling CVF-bound C5 (Supplementary Figure S5). Superimposing this particular conformation of bovine $\mathrm{C} 3$ on $\mathrm{C} 5$ in our C5-CVFBb model allows us to investigate how $\mathrm{C} 3$ may bind to CVFBb (Figure 5B). In comparison with the C5CVFBb model, the scissile peptide bond Arg746-Ser747 in bovine C3 (Figure 5E) is apparently free to reach the catalytic site. Hence, fewer local conformational changes around the 
scissile bond would be required in C3 compared with $\mathrm{C} 5$ after binding to $\mathrm{CVFBb}$, which is consistent with the $\approx 100$-fold faster turnover of $\mathrm{C} 3$ compared with $\mathrm{C} 5$ for $\mathrm{CVFBb}$ and $\mathrm{C} 3 \mathrm{bBb}$ (Rawal and Pangburn, 2000).

In conclusion, the derived $\mathrm{C} 5-\mathrm{CVFBb}$ model suggests that CVF binding places the C5 MG7 and C5a domains more or less correctly relative to $\mathrm{Bb}$, which might explain why $\mathrm{Bb}$ contributes little to CVFBb affinity for C5 (Rawal and Pangburn, 2000). Modest overall repositioning of Bb relative to $\mathrm{C} 5$ in combination with local conformational changes in C5 residues 741-769 and the $\mathrm{Bb}$ loops facing $\mathrm{C} 5$ are then probably sufficient to place Arg751 in the Bb catalytic site possibly combined with a movement of $\mathrm{C} 5 \mathrm{a} \alpha$ helix 1 .

\section{The endogenous $C 3$ and C5 convertases}

In terms of sequence homology, CVF is $49 \%$ identical to human C3 compared with, for example, $77 \%$ sequence identity between human and bovine $\mathrm{C} 3$. In combination with the functional and structural similarities between CVF and $\mathrm{C} 3 \mathrm{~b}$, we expect that $\mathrm{C} 3 \mathrm{~b}$ in the AP convertases binds the substrates (C3 and C5) in a manner analogously to how CVF recognizes $\mathrm{C} 5$. By further extrapolation-which is justified by the pronounced functional similarities between the AP and the $\mathrm{CP}$ convertases-we also expect this to apply to $\mathrm{C} 4 \mathrm{~b}$ in the $\mathrm{CP}$ convertases. We therefore propose a general model for substrate-convertase recognition applicable to both $\mathrm{C} 3$ and C5 convertases. In this model, the MG4-MG5 domains of $\mathrm{C} 3 \mathrm{~b} / \mathrm{C} 4 \mathrm{~b}$ interact with the MG4-MG5 domains of the substrates $\mathrm{C} 3$ and $\mathrm{C} 5$ at one interface (the MG4-MG5 interface), while the MG6-MG7 domains of $\mathrm{C} 3 \mathrm{~b} / \mathrm{C} 4 \mathrm{~b}$ interact with the substrate MG7 domain at a second interface (the MG7 interface). This proposal is compatible with a variety of experimental results.

With respect to the AP C3 convertase, C3b-C3b interactions mediated by the MG4 and MG5 domains are present in the $\mathrm{C} 3 \mathrm{~b}-\mathrm{Bb}-\mathrm{SCIN}$ structure, which strongly resemble the MG4-MG5 interface observed in the C5-CVF structure (Supplementary Figure S8), and these interactions have already been suggested to mirror convertase-C3 interactions (Rooijakkers et al, 2009). The involvement of the MG4-MG5 interface in convertase-substrate interactions is in agreement with the inhibitory effects and structural data on compstatin (Janssen et al, 2007) and CRIg (Wiesmann et al, 2006), as these would prevent substrate binding to the AP convertase due to steric hindrance (Supplementary Figure S7B). Likewise, the involvement of the C3b MG4 domain in substrate recognition consistently explains the effects upon mutation of $\mathrm{C} 3$ Met373 to a threonine, which leads to AP dysfunction (Sfyroera et al, 2010). At the substrate level, the C3 Met373 mutation leads to reduced C3 cleavage by the AP convertase, in agreement with the C3 MG4 domain being involved in $\mathrm{C} 3 \mathrm{~b}$ recognition. In both $\mathrm{C} 3$ and $\mathrm{C} 3 \mathrm{~b}, \mathrm{Met} 373$ (corresponding to CVF Thr363) is located in a $\beta$ strand following a loop region, where the corresponding loops in C5 and CVF appear to participate in intermolecular MG4 and MG5 interactions (Supplementary Figure S2A and C). Our general model also predicts that in the initiating AP convertase $\mathrm{C} 3\left(\mathrm{H}_{2} \mathrm{O}\right) \mathrm{Bb}$, the uncleaved $\mathrm{Nt}-\alpha^{\prime}$ and the $\mathrm{C} 3 \mathrm{a}$ domain are likely to be exposed in proximity to the MG7 domain of the substrate. This is in agreement with the ability of polyclonal C3a-specific antibodies to inhibit this convertase (Pangburn et al, 1981), probably through steric hindrance between the antibodies and the substrate. Concerning the CP C5 convertase, our model predicts that the C4b MG4 and MG5 domains interact with the C5 MG4 and MG5 domains, which is in excellent agreement with the importance of $\mathrm{C} 4 \mathrm{~b}$ residues centred on Arg481 (Supplementary Figure S9) for recognition of C5 by the convertase (Ebanks and Isenman, 1995).

A second important prediction made by our general model is that $\mathrm{C} 3 \mathrm{~b} / \mathrm{C} 4 \mathrm{~b}$ interacts with the substrates $\mathrm{C} 3$ and $\mathrm{C} 5$ through the MG7 interface as observed in the C5-CVF structure. For the AP convertase, $\mathrm{C} 3 \mathrm{~b}$ recognition of the MG7 domain of substrate C3 is in accordance with the data on CRIg (Wiesmann et al, 2006) and the S77 antibody (Katschke et al, 2009) (Supplementary Figure S7B) and explains the failure of the AP C3 convertase to cleave the C3 mutant C3923 DDG where Asp923-Gly924 are deleted (Martinez-Barricarte et al, 2010). Residues 923-924 are located in the MG7 domain right after the stretch Ala915Ser921, which our general model predicts to be in contact with the convertase by comparison with C5 (Supplementary Figure S2B). An interaction of the substrate MG7 domain with $\mathrm{C} 4 \mathrm{~b}$ in $\mathrm{CP}$ convertases agrees with the deletion of $\mathrm{C5}$ residues Ser881-Gln886, of which four contact CVF (Figure 3E and F; Supplementary Figure S2A), and reduce haemolytic activity through the $\mathrm{CP}$ to $<10 \%$ (Low et al, 1999).

In our C5-CVF structure the C5 C345C domain is positioned far away from CVF, but peptides containing $\mathrm{C} 5$ residues 1628-1633 and mutations in this region inhibit C5 cleavage by the CP C5 convertase (Sandoval et al, 2000). This is in apparent conflict with our hypothesis that C4b as a subunit of the $\mathrm{CP}$ C5 convertase and $\mathrm{CVF}$ will recognize $\mathrm{C} 5$ in a similar manner. By rotating the $\mathrm{C} 5 \mathrm{C} 345 \mathrm{C}$ domain in our C5-CVF-Bb model around the Cys866-Cys1527 disulphide bridge as in free C5 (Fredslund et al, 2008), however, the C5 region 1628-1633 becomes located close to the CVF Nt- $\gamma$ chain and $\mathrm{Bb}$ (Supplementary Figure S10). Hence, upon binding to the $\mathrm{CP}$ C5 convertase, the $\mathrm{C} 5 \mathrm{C} 345 \mathrm{C}$ domain is possibly located close to $\mathrm{C} 2 \mathrm{a}$ and the $\mathrm{C} 4 \mathrm{~b} \mathrm{Nt}-\alpha^{\prime}$. Rotation of the $\mathrm{C} 345 \mathrm{C}$ domain in this manner could also explain how the tick protein OmCI inhibits $\mathrm{C} 5$ cleavage by stabilizing an exposed position of the $\mathrm{C} 345 \mathrm{C}$ domain (Fredslund et al, 2008). As C5 cleavage by CVFBb is inhibited neither by the $\mathrm{C} 5$ peptides nor by mutations inhibiting the $\mathrm{CP}$ C5 convertase (Sandoval et al, 2000), the suggested contacts between the C5 $\mathrm{C} 345 \mathrm{C}$ domain and the convertase subunits might be specific to the CP C5 convertase.

Collectively, our structure and numerous experimental observations suggest that $\mathrm{C} 3 \mathrm{~b}$ and $\mathrm{C} 4 \mathrm{~b}$ in the endogenous convertases recognize both $\mathrm{C} 3$ and $\mathrm{C} 5$ similarly to how $\mathrm{CVF}$ recognizes $\mathrm{C} 5$. In particular, we can explain data relating to both AP and CP convertases and to both substrates C3 and C5. Our proposal that the convertases interact with the substrate through the MG4-MG5 interface and the MG7 interface also agrees with prior experimental results for both $\mathrm{AP}$ and $\mathrm{CP}$ convertases.

\section{Discussion}

C5-CVF as a model for substrate-convertase complexes Proteolysis of complement components C3 and C5 by the convertases leads to opsonization, pathogen lysis through MAC assembly, chemotaxis and stimulation of the adaptive immune system, and is thus crucial for the effector functions 
of the complement system (Walport, 2001; Gasque, 2004). Structural knowledge concerning recognition of the substrate by the convertase enzymes is essential for the development of selective complement inhibitors. Because of the size and very limited lifetime of the endogenous convertases, co-crystals with their substrates are most likely technically difficult to generate, especially for the C5 convertases. Alternatively, substrate-convertase complexes may be studied by electron microscopy at rather low resolution, and currently the best EM reconstruction of a convertase has a resolution of $\sim 27 \AA$ (Torreira et al, 2009). Homology in terms of sequence, structure, and function between CVF and C3b/C4b suggests that our C5-CVF structure provides a general model for how the complement convertases recognize their substrate. Assuming that $\mathrm{C} 3 \mathrm{~b} / \mathrm{C} 4 \mathrm{~b}$ in the endogenous convertases recognizes the substrates similarly to how CVF recognizes $\mathrm{C} 5$, we can rationalize a large number of experimental results concerning these convertases. Based on the C3b-C3b interactions in the structure of $\mathrm{C} 3 \mathrm{~b}-\mathrm{Bb}-\mathrm{SCIN}$, it was already suggested that the MG4-MG5 domains in C3b/C4b in the convertases might interact with the MG4-MG5 domains in $\mathrm{C} 3$, whereas it was questioned whether C5 could be recognized by the convertases in the same manner (Rooijakkers et al, 2009). Our C5-CVF structure strongly argues that the MG4-MG5 interface is entirely universal no matter what substrate or convertase is considered. In addition, the C3b$\mathrm{Bb}-\mathrm{SCIN}$ structure did not reveal the recognition of the substrate MG7 domain present in the C5-CVF complex. Mutation studies strongly propose that this part of the $\mathrm{C} 5-$ CVF interface is conserved when C5 interacts with the CP C5 convertases (Low et al, 1999) and when C3 is recognized by the AP C3 convertase (Martinez-Barricarte et al, 2010). Collectively, the C5-CVF structure, together with previous data on the endogenous convertases, suggests that substrateconvertase interactions through the MG4-MG5 interface and the MG7 interface are a conserved feature common among convertases. Our model of the CVFBb-C5 complex suggests that the conformational changes imposed by CVF on C5 place its cleavage site Arg751-Leu752 in proximity to the catalytic triad in $\mathrm{Bb}$, and that subsequently only minor local changes are necessary in $\mathrm{C} 5$ and $\mathrm{Bb}$ to accommodate Arg751 of C5 in the active site of $\mathrm{Bb}$. Although the resolution of our crystal structure is only $4.3 \AA$, it provides the first structural insight into how the substrate is recognized by the convertases.

\section{The conformational change in nascent $\mathbf{C} 3 \mathrm{~b}$}

Upon cleavage of $\mathrm{C} 3$ to $\mathrm{C} 3 \mathrm{~b}$, dramatic conformational changes especially within the $\mathrm{C} 3 \mathrm{~b} \alpha^{\prime}$ chain occur (Janssen et al, 2006; Wiesmann et al, 2006). In particular, the reactive thioester of nascent $\mathrm{C} 3 \mathrm{~b}$ becomes exposed and moves by $>80 \AA$. Based on C3b-C3b interactions in the C3b-Bb-SCIN structure (Rooijakkers et al, 2009), it was suggested that the MG4 and MG5 domains of nascent C3b can remain bound to the convertase, while the TED, CUB, MG7, and MG8 domains rearrange to the $\mathrm{C} 3 \mathrm{~b}$ conformation. Our general substrateconvertase model allows us to investigate the post-cleavage conformational change occurring in nascent C3b (Supplementary Figure S11A; Supplementary Animation 2), and this simulation supports that nascent $\mathrm{C} 3 \mathrm{~b}$ remains in contact with the convertase throughout the conformational rearrangement. A conformational change like that from C3 to $\mathrm{C} 3 \mathrm{~b}$ is expected to occur at a timescale of $10-1000 \mu$ s (Adcock and McCammon, 2006). The thioester in nascent C3b has a lifetime of $\approx 60 \mu$ s with an attachment efficiency of only $10 \%$ (Law and Dodds, 1997); consequently, quick attachment to the activating surface is essential. Based on our model of the substrate-convertase complex with a head-to-head arrangement of the substrate and the $\mathrm{C} 3 \mathrm{~b} / \mathrm{C} 4 \mathrm{~b}$ in the convertase, an orientation of the surface-bound $\mathrm{C} 3$ convertases, where the long axis of the convertase $\mathrm{C} 3 \mathrm{~b} / \mathrm{C} 4 \mathrm{~b}$ is roughly perpendicular to the surface, appears optimal in minimizing thioester hydrolysis in nascent C3b before surface attachment. In this orientation, the thioester of nascent $\mathrm{C} 3 \mathrm{~b}$ will move directly towards the same surface where the convertase $\mathrm{C} 3 \mathrm{~b} / \mathrm{C} 4 \mathrm{~b}$ is attached (Supplementary Figure S11A; Supplementary Animation 2). If the nascent $\mathrm{C} 3 \mathrm{~b}$ remains associated with the convertase during the conformational change in a product-enzyme complex, this will both direct the reactive thioester during the conformational change to the surface and minimize the diffusion of nascent $\mathrm{C} 3 \mathrm{~b}$ from the convertase. Besides decreasing the efficiency of thioester attachment, diffusion of nascent $\mathrm{C} 3 \mathrm{~b}$ could lead to delocalization of the complement response, and, in the worst case, cause attachment of $\mathrm{C} 3 \mathrm{~b}$ to host cells.

\section{The CP C5 convertase}

The high $K_{\mathrm{m}}$ value of the $\mathrm{C} 3$ convertases for $\mathrm{C} 5$ implies that the convertases will primarily cleave C3 and not C5 at their physiological concentrations (Rawal and Pangburn, 2000; Rawal et al, 2008). Covalent binding or non-covalent association of a C3b molecule to the $\mathrm{C} 3$ convertases changes the substrate specificity from C3 to C5 and decreases the $K_{\mathrm{m}}$ value for C5 100-1000-fold (Rawal and Pangburn, 2000; Rawal et al, 2008), but whether this C3b molecule directly interacts with C5 or exerts its effect solely through contacts with the C3 convertase subunits is currently unknown. C3b becomes covalently attached to either $\mathrm{C} 3$ convertase through the nascent thioester. In the CP C5 convertase, Ser1236 in C4b is the nucleophile attacking the thioester in nascent $\mathrm{C} 3 \mathrm{~b}$, but crosslinking is not essential for CP C5 convertase activity (Kim et al, 1992). The linear relationship between the number of C4b$\mathrm{C} 3 \mathrm{~b}$ dimers and the number of high-affinity C5 binding sites (Takata et al, 1987) suggest that the cross-linked C3b acts in cis and increases the $\mathrm{C} 5$ affinity of the $\mathrm{C} 4 \mathrm{~b}$ it is covalently bound to. The cross-link between the $\mathrm{C} 3 \mathrm{~b}$ thioester glutamine and Ser1236 in C4b provides a simple distance criterion that gives a rough 'maximum' estimate of how various parts of C3b could interact with $\mathrm{C} 4 \mathrm{~b}, \mathrm{C} 2 \mathrm{a}$ and the $\mathrm{C} 5$ substrate to shift the specificity from $\mathrm{C} 3$ to $\mathrm{C} 5$. Assuming that $\mathrm{C} 3 \mathrm{~b}$ when crosslinked to $\mathrm{C} 4 \mathrm{~b}$ adopts a conformation similar to that of known structures of C3b, the MG1, MG4, MG5, and TED domains of C3b will be able to contact the CUB and TED domains of C4b. The CUB, MG2, MG6, and MG3 domains appear to be capable of reaching mainly the rest of $\mathrm{C} 4 \mathrm{~b}$, while the MG7, MG8, and C345C domains potentially could be in direct contact with C5 (Supplementary Figure S11B).

\section{Complement inhibition}

S. aureus secretes a diverse repertoire of proteins as part of its immune evasion strategy. By employing the bifunctional SSL7, S. aureus simultaneously manipulates two important branches of the immune system. Full inhibition of C5 cleavage by SSL7 is dependent on IgA, no matter if the endogenous convertases or CVFBb is studied (Bestebroer et al, 2010; 
Laursen et al, 2010). The structure of SSL7-C5-CVF confirms that binding of SSL7 alone is not sufficient to prevent binding of $\mathrm{C} 5$ to $\mathrm{CVF}$, and thereby completely inhibit cleavage by $\mathrm{CVFBb}$. Although details of substrate recognition will certainly be different for the endogenous convertases, CVF most likely mimics $\mathrm{C} 3 \mathrm{~b}$ in the AP $\mathrm{C} 5$ convertase and $\mathrm{C} 4 \mathrm{~b}$ in the $\mathrm{CP}$ C5 convertase. This is supported by the observation that SSL7 only marginally changes the interaction between $\mathrm{C} 5$ and $\mathrm{C} 3 \mathrm{~b}$. However, as the conformation and location of the second $\mathrm{C} 3 \mathrm{~b}$ molecule in the C5 convertase are unknown, steric hindrance between SSL7 and the second C3b molecule cannot be excluded either.

Deciphering the molecular mechanisms of immune evasion is important for understanding the pathogenesis and may help in developing more effective therapeutic strategies. Furthermore, we may seek inspiration to develop specific complement inhibitors aiming at controlling the complement under different pathological conditions in learning from $S$. aureus and other organisms. In paroxysmal nocturnal haemoglobinuria, MAC deposition through C5b causes haemolysis of erythrocytes (Rother et al, 2007). We have previously suggested that targeting the SSL7-binding region on C5 could be used to selectively block MAC formation without affecting the role of $\mathrm{C} 5 \mathrm{a}$ in regulating innate immunity (Laursen et al, 2010). Our structure of the C5-CVF complex and the very recent structure of C5b6 (Hadders et al, 2010) offer novel information on potential target regions in the $\mathrm{C} 5$ molecule allowing C5 cleavage but preventing MAC formation. Epitopes for C5 antibodies can now be chosen such that the antibody does not interfere with $\mathrm{C} 5$ cleavage, and, by comparison with the C5bC6 structure, C5 epitopes that have an important role in $\mathrm{C} 6$ binding or are involved in stabilization of the C5b conformation can be pinpointed.

In summary, our structure of the C5-CVF complex provides for the first time structural insight into substrate recognition by the complement convertases. Our ability to rationalize prior experimental results obtained in studies of the endogenous convertases shows that the C5-CVF structure provides a guide for understanding how these intricate proteolytic enzymes recognize their substrates C3 and C5.

\section{Materials and methods}

\section{Protein purification and crystallization}

Human C5, CVF (Naja kaouthia), and SSL7 (strain ATCC 12598) were purified as described (Fredslund et al, 2008; Laursen et al, 2010). Before crystallization, the proteins were concentrated and mixed in 1:1 molar ratio to a final concentration of $25 \mu \mathrm{M}$. Crystals were grown at $4{ }^{\circ} \mathrm{C}$ in vapour diffusion experiments by mixing equal volumes of protein with a reservoir solution containing $1.1-1.3 \mathrm{M}$ sodium malonate, $\mathrm{pH}$ 7.4. The crystals were cryo-protected by equilibration in $3 \mathrm{M}$ sodium malonate, $\mathrm{pH} 7.4$, before freezing in liquid nitrogen.

\section{Structure determination and analysis}

Diffraction data (Table I) collected at ESRF ID14-4 at $\lambda=0.9765 \AA$ (C5-CVF) or ESRF ID29 at $\lambda=0.9763 \AA$ (SSL7-C5-CVF) were processed and scaled using XDS and XSCALE (Kabsch, 2001). Both structures were solved by molecular replacement using PHENIX (Adams et al, 2002). The C5-CVF structure was solved using the C5 domains C5d, CUB, and MG8 (residues 932-1514), and CVF from the CVFB structure (RCSB entry 3HRZ) with the C-terminal C345C domain omitted as two independent search models. The remaining domains of $\mathrm{C} 5$ and $\mathrm{CVF}$ were subsequently placed manually into a $2 \mathrm{mF}_{\mathrm{o}}-\mathrm{DF}_{\mathrm{c}}$ electron density map using the graphics program ' $\mathrm{O}$ ' (Jones et al, 1991). The SSL7-C5-CVF structure was solved by molecular replacement using CVF without the C345C domain and the C5d, CUB, and MG8 domains in C5 from an intermediate C5-CVF structure as a search model in molecular replacement, after which the remaining domains were docked into a $2 \mathrm{mF}_{\mathrm{o}}-\mathrm{DF}_{\mathrm{c}}$ electron density map. From this point, multiple crystal averaging with DM (Cowtan, 1994) provided improved solvent flattened and averaged maps, which were used during rebuilding in combination with $2 \mathrm{mF}_{\mathrm{o}}-\mathrm{DF}_{\mathrm{c}}$ electron density maps. Alternatively, real-space averaging of $2 \mathrm{mF}_{\mathrm{o}}-\mathrm{DF}_{\mathrm{c}}$ electron density maps from PHENIX.REFINE was done with AVE (Kleywegt and Jones, 1994). Both structures were rebuilt in ' $\mathrm{O}$ ' and refined using PHENIX.REFINE with tight non-crystallographic restraints between the two copies of the complexes present in the asymmetric unit and MLHL restraints derived from the multiple crystal averaging. Structure quality was assessed with PROCHECK (Laskowski et al, 1993). In the final C5-CVF structure, $74.5 \%$ of the residues had a main-chain conformation in the most favoured regions, $24.7 \%$ in allowed regions, and $0.9 \%$ in disallowed regions of the Ramachandran plot. For the SSL7-C5-CVF structure the corresponding numbers were $72.3,26.7$ and $1.0 \%$, respectively. Conformational changes were analysed with DYNDOM (Hayward and Berendsen, 1998) and intermolecular interfaces with PISA (Krissinel and Henrick, 2007). Molecular graphics figures were created in PYMOL (DeLano, 2002), which was also used for superposition of structures and creation of animations with the RIGIMOL plug-in. The electrostatic potential was plotted on the solvent-accessible surface with the APBS (Baker et al, 2001) plug-in for PYMOL with a gradient from $-10 \mathrm{kT} / \mathrm{e}$ (red) to $+10 \mathrm{kT} / \mathrm{e}$ (blue). Charge and radius parameters according to an AMBER force field were assigned to the PDB file by the PDB2PQR web server (Dolinsky et al, 2004).

\section{SPR measurements}

Human C3b was generated as described (Wu et al, 2009) and further purified on a Source $15 \mathrm{~S}(9 \mathrm{ml}$, GE Healthcare) column equilibrated in $20 \mathrm{~mm}$ MES, pH 6.0. C3b was eluted with a linear gradient of $0-500 \mathrm{~mm} \mathrm{NaCl}$ over 20 column volumes. Fractions containing C3b were concentrated and further purified over a Superdex 200 HR 10/30 column (GE Healthcare). All SPR experiments were performed on a BIACORE T100 instrument at $25^{\circ} \mathrm{C}$, using a CM5 sensor chip with amine-coupled SSL7 (4000 resonance units). A constant flow rate of $30 \mu \mathrm{l} / \mathrm{min}$ was used and all experiments were performed in a buffer containing $150 \mathrm{mM} \mathrm{NaCl}$, $10 \mathrm{mM}$ Hepes, $\mathrm{pH}$ 7.4, $50 \mu \mathrm{M}$ EDTA, and $10 \mathrm{mM} \mathrm{MgCl}_{2}$. Regeneration was done in the same buffer after addition of $500 \mathrm{mM} \mathrm{NaCl}$. Before each individual measurement, immobilized SSL7 was saturated with C5 $(100 \mathrm{nM})$. Binding affinities of CVF and C3b to SSL7-C5 were determined by global fitting to a 1:1 binding model or a model assuming 1:1 binding in combination with a conformational change using the BIACORE evaluation program.

\section{Accession numbers}

Coordinates and structure factors for the SSL7-C5-CVF and C5-CVF complexes are deposited at the RCSB protein data bank as entries 3PRX and 3PVM, respectively.

\section{Supplementary data}

Supplementary data are available at The EMBO Journal Online (http://www.embojournal.org).

\section{Acknowledgements}

We are grateful to the staff at ESRF and SLS beamlines for help with data collection, L Kristensen for protein purification, and S Thiel for discussions. GRA was supported by the Science Research Council FNU, the Lundbeck Foundation (LUNA), and a Hallas-Møller stipend from the Novo-Nordisk Foundation.

Author contributions: NSL: purification, crystallization, data collection, structure determination and analysis, and manuscript preparation; KRA: purification, SPR data collection, and data analysis; IB: purification; ES: purification and analysis; LSJ: purification, analysis, and study design; GRA: study design, analysis, and manuscript preparation.

\section{Conflict of interest}

The authors declare that they have no conflict of interest. 


\section{References}

Adams PD, Grosse-Kunstleve RW, Hung LW, Ioerger TR, McCoy AJ, Moriarty NW, Read RJ, Sacchettini JC, Sauter NK, Terwilliger TC (2002) PHENIX: building new software for automated crystallographic structure determination. Acta Crystallogr D Biol Crystallogr 58: 1948-1954

Adcock SA, McCammon JA (2006) Molecular dynamics: survey of methods for simulating the activity of proteins. Chem Rev 106: $1589-1615$

Baker NA, Sept D, Joseph S, Holst MJ, McCammon JA (2001) Electrostatics of nanosystems: application to microtubules and the ribosome. Proc Natl Acad Sci USA 98: 10037-10041

Bestebroer J, Aerts PC, Rooijakkers SH, Pandey MK, Kohl J, van Strijp JA, de Haas CJ (2010) Functional basis for complement evasion by staphylococcal superantigen-like 7. Cell Microbiol 12: 1506-1516

Cook WJ, Galakatos N, Boyar WC, Walter RL, Ealick SE (2010) Structure of human desArg-C5a. Acta Crystallogr D Biol Crystallogr 66: 190-197

Cowtan K (1994) 'Dm': an automated procedure for phase improvement by density modification. Joint CCP4 and ESF-EACBM Newsletter on Protein Crystallography 31: 34-38

DeLano WL (2002) The PyMOL User's Manual. San Carlos, CA, USA: DeLano Scientific

Dolinsky TJ, Nielsen JE, McCammon JA, Baker NA (2004) PDB2PQR: an automated pipeline for the setup of PoissonBoltzmann electrostatics calculations. Nucleic Acids Res 32: W665-W667

Ebanks RO, Isenman DE (1995) Evidence for the involvement of arginine 462 and the flanking sequence of human $\mathrm{C} 4$ beta-chain in mediating $\mathrm{C} 5$ binding to the $\mathrm{C} 4 \mathrm{~b}$ subcomponent of the classical complement pathway C5 convertase. J Immunol 154: 2808-2820

Fredslund F, Jenner L, Husted LB, Nyborg J, Andersen GR, SottrupJensen L (2006) The structure of bovine complement component 3 reveals the basis for thioester function. J Mol Biol 361: 115-127

Fredslund F, Laursen NS, Roversi P, Jenner L, Oliveira CL, Pedersen JS, Nunn MA, Lea SM, Discipio R, Sottrup-Jensen L, Andersen GR (2008) Structure of and influence of a tick complement inhibitor on human complement component 5. Nat Immunol 9: 753-760

Gasque P (2004) Complement: a unique innate immune sensor for danger signals. Mol Immunol 41: 1089-1098

Geisbrecht BV (2008) Staphylococcal complement inhibitors: biological functions, recognition of complement components, and potential therapeutic implications. Adv Exp Med Biol 632: 221-236

Hadders MA, Pangburn MK, Svergun DI, Gros P (2010) Structural insights into the initiation of the terminal pathway. Mol Immunol 47: 2257-2258

Hayward S, Berendsen HJ (1998) Systematic analysis of domain motions in proteins from conformational change: new results on citrate synthase and T4 lysozyme. Proteins 30: 144-154

Janssen BJ, Christodoulidou A, McCarthy A, Lambris JD, Gros P (2006) Structure of C3b reveals conformational changes that underlie complement activity. Nature 444: 213-216

Janssen BJ, Gomes L, Koning RI, Svergun DI, Koster AJ, Fritzinger DC, Vogel CW, Gros P (2009) Insights into complement convertase formation based on the structure of the factor B-cobra venom factor complex. EMBO J 28: 2469-2478

Janssen BJ, Halff EF, Lambris JD, Gros P (2007) Structure of compstatin in complex with complement component $\mathrm{C} 3 \mathrm{c}$ reveals a new mechanism of complement inhibition. J Biol Chem 282: 29241-29247

Janssen BJ, Huizinga EG, Raaijmakers HC, Roos A, Daha MR, Nilsson-Ekdahl K, Nilsson B, Gros P (2005) Structures of complement component $\mathrm{C} 3$ provide insights into the function and evolution of immunity. Nature 437: 505-511

Jones TA, Cowan S, Zou J-Y, Kjeldgaard M (1991) Improved methods for building protein models in electron density maps and the location of errors in these models. Acta Cryst A 47: 110-119

Kabsch W (2001) XDS. In International Tables for Crystallography, Crystallography of Biological Macromolecules, Rossmann MG, Arnold E (eds), Vol. F, p Chapter 25.22.29. Dordrecht: Kluwer Academic Publishers

Katschke Jr KJ, Stawicki S, Yin J, Steffek M, Xi H, Sturgeon L, Hass PE, Loyet KM, Deforge L, Wu Y, van Lookeren Campagne M,
Wiesmann C (2009) Structural and functional analysis of a C3bspecific antibody that selectively inhibits the alternative pathway of complement. J Biol Chem 284: 10473-10479

Kim YU, Carroll MC, Isenman DE, Nonaka $M$, Pramoonjago $P$, Takeda J, Inoue K, Kinoshita T (1992) Covalent binding of C3b to $\mathrm{C} 4 \mathrm{~b}$ within the classical complement pathway $\mathrm{C} 5$ convertase. Determination of amino acid residues involved in ester linkage formation. J Biol Chem 267: 4171-4176

Kinoshita T, Takata Y, Kozono H, Takeda J, Hong KS, Inoue K (1988) C5 convertase of the alternative complement pathway: covalent linkage between two $\mathrm{C} 3 \mathrm{~b}$ molecules within the trimolecular complex enzyme. J Immunol 141: 3895-3901

Kleywegt GJ, Jones TA (1994) Halloween... masks and bones. In From First Map to Final Model, Bailey S, Hubbard R, Waller D (eds), pp 59-66. Warrington: SERC Daresbury Laboratory

Krishnan V, Ponnuraj K, Xu Y, Macon K, Volanakis JE, Narayana SV (2009) The crystal structure of cobra venom factor, a cofactor for C3- and C5-convertase CVFBb. Structure 17: 611-619

Krissinel E, Henrick K (2007) Inference of macromolecular assemblies from crystalline state. J Mol Biol 372: 774-797

Langley R, Wines B, Willoughby N, Basu I, Proft T, Fraser JD (2005) The staphylococcal superantigen-like protein 7 binds IgA and complement C5 and inhibits IgA-Fc alpha RI binding and serum killing of bacteria. J Immunol 174: 2926-2933

Laskowski RA, MacArthur MW, Moss D, Thornton JM (1993) PROCHECK: a program to check the stereochemical quality of protein structures. J Appl Cryst 26: 283-291

Laursen NS, Gordon N, Hermans S, Lorenz N, Jackson N, Wines B, Spillner E, Christensen JB, Jensen M, Fredslund F, Bjerre M, Sottrup-Jensen L, Fraser JD, Andersen GR (2010) Structural basis for inhibition of complement C5 by the SSL7 protein from Staphylococcus aureus. Proc Natl Acad Sci USA 107: 3681-3686

Law SK, Dodds AW (1997) The internal thioester and the covalent binding properties of the complement proteins $\mathrm{C} 3$ and $\mathrm{C} 4$. Protein Sci 6: $263-274$

Low PJ, Ai R, Ogata RT (1999) Active sites in complement components $\mathrm{C} 5$ and $\mathrm{C} 3$ identified by proximity to indels in the $\mathrm{C} 3 / 4 / 5$ protein family. J Immunol 162: 6580-6588

Martinez-Barricarte $\mathrm{R}$, Heurich $\mathrm{M}$, Valdes-Canedo $\mathrm{F}$, VazquezMartul E, Torreira E, Montes T, Tortajada A, Pinto S, LopezTrascasa M, Morgan BP, Llorca O, Harris CL, Rodriguez de Cordoba S (2010) Human C3 mutation reveals a mechanism of dense deposit disease pathogenesis and provides insights into complement activation and regulation. J Clin Invest 120: 3702-3712

Pangburn MK, Muller-Eberhard HJ (1983) Initiation of the alternative complement pathway due to spontaneous hydrolysis of the thioester of C3. Ann NY Acad Sci 421: 291-298

Pangburn MK, Rawal N (2002) Structure and function of complement C5 convertase enzymes. Biochem Soc Trans 30: 1006-1010

Pangburn MK, Schreiber RD, Muller-Eberhard HJ (1981) Formation of the initial C3 convertase of the alternative complement pathway. Acquisition of C3b-like activities by spontaneous hydrolysis of the putative thioester in native C3. J Exp Med 154: 856-867

Ramsland PA, Willoughby N, Trist HM, Farrugia W, Hogarth PM, Fraser JD, Wines BD (2007) Structural basis for evasion of IgA immunity by Staphylococcus aureus revealed in the complex of SSL7 with Fc of human IgA1. Proc Natl Acad Sci USA 104: $15051-15056$

Rawal N, Pangburn MK (2000) Functional role of the noncatalytic subunit of complement C5 convertase. J Immunol 164: $1379-1385$

Rawal N, Rajagopalan R, Salvi VP (2008) Activation of complement component C5: comparison of C5 convertases of the lectin pathway and the classical pathway of complement. J Biol Chem 283: 7853-7863

Rooijakkers SH, Wu J, Ruyken M, van Domselaar R, Planken KL, Tzekou A, Ricklin D, Lambris JD, Janssen BJ, van Strijp JA, Gros P (2009) Structural and functional implications of the alternative complement pathway $\mathrm{C} 3$ convertase stabilized by a staphylococcal inhibitor. Nat Immunol 10: 728-733

Rother RP, Rollins SA, Mojcik CF, Brodsky RA, Bell L (2007) Discovery and development of the complement inhibitor eculizumab for the treatment of paroxysmal nocturnal hemoglobinuria. Nat Biotechnol 25: 1256-1264 
Sandoval A, Ai R, Ostresh JM, Ogata RT (2000) Distal recognition site for classical pathway convertase located in the $\mathrm{C} 345 \mathrm{C} /$ netrin module of complement component C5. J Immunol 165: 1066-1073

Sfyroera G, Ricklin D, Chen H, Nilsson-Ekdahl K, Nilsson B, Wu EL, Kaznessis YN, Lambris JD (2010) Impaired ability of complement activation via the alternative pathway $\mathrm{C} 3$ convertase caused by a single-point mutation in the beta chain of C3. Mol Immunol 47: 2289-2289

Takata Y, Kinoshita T, Kozono H, Takeda J, Tanaka E, Hong K, Inoue K (1987) Covalent association of C3b with C4b within C5 convertase of the classical complement pathway. J Exp Med 165: $1494-1507$

Torreira E, Tortajada A, Montes T, Rodriguez de Cordoba S, Llorca O (2009) 3D structure of the C3bB complex provides insights into the activation and regulation of the complement alternative pathway convertase. Proc Natl Acad Sci USA 106: 882-887

Vogel CW, Fritzinger DC (2010) Cobra venom factor: structure, function, and humanization for therapeutic complement depletion. Toxicon 56: 1198-1222

Walport MJ (2001) Complement. First of two parts. N Engl J Med 344: 1058-1066

Wiesmann C, Katschke KJ, Yin J, Helmy KY, Steffek M, Fairbrother WJ, McCallum SA, Embuscado L, DeForge L, Hass PE, van Lookeren Campagne M (2006) Structure of C3b in complex with CRIg gives insights into regulation of complement activation. Nature 444: 217-220

Wu J, Wu YQ, Ricklin D, Janssen BJ, Lambris JD, Gros P (2009) Structure of complement fragment C3b-factor $\mathrm{H}$ and implications for host protection by complement regulators. Nat Immunol 10: 728-733 